\section{CARACTERÍSTICAS DOS ACIDENTES COM MATERIAIS BIOLÓGICOS POR ENFERMEIRAS E ENFERMEIROS NO ESTADO DA BAHIA, BRASIL}

\section{CHARACTERISTICS OF ACCIDENTS WITH BIOLOGICAL MATERIALS BY NURSES IN BAHIA STATE, BRAZIL}

Iara Caroline Moura Conceição da Silva ${ }^{1} /$ Ludilvania Almeida Silva ${ }^{1}$ / Marcela Andrade Rios ${ }^{2}$ / Rebeca de Jesus Silva ${ }^{1, *} /$ Rubia Pinto Carvalho ${ }^{1 /}$ Sabrina Alves Nunes ${ }^{1}$

\section{INTRODUÇÃO}

Profissionais da saúde, sejam socorristas ou de assistência domiciliar são frequentemente expostos à fluidos orgânicos e sangue. Acidentes com estes materiais biológicos são regulares nos ambientes de trabalho se tornando um potencial risco de contaminação. Incidentes ocasionados com materiais perfuro-cortantes são capazes de transmitir mais de 20 patógenos diferentes. A imunização e o atendimento correto pós-exposição são fundamentais diante de um acidente ocupacional (MINISTÉRIO DA SAUDE, 2006).

De acordo com o Ministério do trabalho, acidente de trabalho se configura como aquele que ocorre pelo exercício do trabalho provocando lesão corporal ou perturbação funcional que cause a morte ou a perda ou redução, permanente ou temporária da capacidade para o trabalho (BRASIL,1991). No âmbito assistencial de saúde é comum a ocorrência de acidentes ocupacionais e, se faz necessário, determinar ações que previnam esses acidentes bem como diagnostico e notificação dos casos ocorridos (MACHADO; MACHADO, 2011).

Segundo o Centers for Disease Control and Prevention (CDC), qualquer fluido corporal pode conter agentes infecciosos, entre eles, o sangue têm sido um dos principais veículos de transmissão do vírus das hepatites $\mathrm{B}$ e $\mathrm{C}$ e do HIV. Tendo a hepatite B grande prevalência entre os profissionais de saúde (MACHADO; MACHADO, 2011).

\section{RESUMO}

Objetivo: Descrever os acidentes com material biológico em enfermeiras e enfermeiros, ocorridos no período compreendido entre os anos de 2009 a 2018, no estado da Bahia. Materiais e Métodos: Trata-se de um estudo descritivo baseados em dados do SINAN - Sistema de Informação de Agravos de Notificação quanto aos acidentes com material biológico em enfermeiras e enfermeiros, no estado da Bahia. Os dados foram analisados por meio do Microsoft Office Excel, com cálculos de frequências absoluta e relativa. Resultados: Foram notificados 1.869 registros de acidentes de trabalho com materiais biológicos por enfermeiras e enfermeiros, sendo a maior frequência encontrada foi no sexo feminino $1.632(87,3 \%)$ com idade entre 20 a 39 anos $(83,5 \%)$, pardas $(52,0 \%)$, com emprego registrado $(36,8 \%)$, o maior índice de contaminação se deu pela forma percutânea $(67,3 \%)$ e o sangue $(77 \%)$ foi o fluido de maior contato para ambos os sexos. Conclusão: se faz necessário medidas preventivas aos acidentes de trabalho em especial na área da saúde, com ações e projetos de educação continuada a esses profissionais.

Palavras-chave: Acidentes de trabalho. Material biológico. Enfermeiro.

\section{ABSTRACT}

Objective: To describe accidents with biological material in nurses, which occurred in the period from 2009 to 2018, in the state oh Bahia. Materials and Methods: This is a descriptive study, based on data from SINAN - Notifiable Diseases Information System about accidents with biological material in nurses from the state of Bahia. The data were analyzed using the Microsoft Office Excel, with calculations of absolute and relative frequencies. Results: 1,869 records of occupational accidents involving biological materials were reported by nurses, the highest frequency was found in females $1,632(87.3 \%)$ aged 20 to 39 years $(83.5 \%)$, brown $(52.0 \%)$, with registered employment $(36.8 \%)$, the highest rate of contamination was due to the percutaneous form $(67.3 \%)$ and blood (77\%) was the fluid of greatest contact for both sexes. Conclusion: preventive measures against occupational accidents are necessary, especially in the health area, with continuing education actions and projects for these professionals.

Keywords: Accidents at work. Biological material. Nurse.

Submetido em: 08 de nov. 2019

Aceito em: 14 de fev. 2020

${ }^{1}$ Universidade do Estado da Bahia - UNEB, Guanambi, Bahia - Brasil.

${ }^{2}$ Universidade Estadual do Sudoeste da Bahia, Jequié, Bahia - Brasil.

${ }^{*}$ E-mail para correspondência: rebecarosaflor@gmail.com 
A exposição aos materiais biológicos pode ocorrer pelas formas percutânea, mucosa e cutânea. Em especial aos profissionais de enfermagem a contaminação ocorre principalmente pela exposição percutânea, sendo o risco de infecção pós-exposição com o vírus do HIV de 0,3\%, a exposição ao vírus de hepatite $\mathrm{B}$ é bem variável podendo chegar a $60 \%$ dependendo do estado do paciente e a hepatite $\mathrm{C}$ o risco de transmissão é de cerca de $2 \%$ pós-exposição (MINISTÉRIO DA SAUDE, 2006).

Muitos acidentes ocorridos com materiais biológicos não são reconhecidos pelos empregadores e trabalhadores como acidente de trabalho e, na maioria das vezes são somente registrados mediante a progressão da doença, dificultando ações de vigilância em saúde do trabalhador (CAIXETA; BARBOSA-BRANCO, 2004).

Diante do exposto o objetivo deste trabalho é descrever os acidentes com material biológico em enfermeiras e enfermeiros, ocorridos no período compreendido entre os anos de 2009 a 2018, no estado da Bahia.

\section{MATERIAIS E MÉTODOS}

Trata-se de um estudo descritivo, realizado com base em dados de notificação e investigação de acidente com material biológico em enfermeiras e enfermeiros, ocorridos no período compreendido entre os anos de 2009 a 2018, no estado da Bahia. Os dados foram obtidos eletronicamente por meio do Sistema de Informações de Agravos de Notificação (SINANNET) através do acesso a Diretoria de Vigilância e Atenção à Saúde do Trabalhador (DIVAST). Foram selecionados os casos de acidentes registrados com o código Z20.9.
Foram selecionadas todas as especialidades de enfermagem disponíveis no sistema, sendo as mesmas: enfermeiro, auditor, de bordo, de centro cirúrgico, de terapia intensiva, do trabalho, nefrologista, neonatologista, obstétrico, psiquiátrico, puericultor e pediátrico, sanitarista, da estratégia de saúde da família e da estratégia de agente comunitário de saúde.

As variáveis analisadas foram divididas em sociodemográficas e vínculo de trabalho (faixa etária, raça/cor, situação no mercado de trabalho e empresa terceirizada) e características do acidente (ano, exposição percutânea, exposição mucosa, material orgânico, uso de equipamento de proteção individual (EPI), fonte conhecida, evolução caso, situação vacina hepatite B e ANTI-HIV).

Os dados foram coletados no mês de setembro de 2019, sendo tabulados e analisados por meio do Microsoft Office Excel, com cálculo das frequências absolutas e relativas.

Por se tratar de dados secundários e de domínio público não foi necessária a submissão ao Comitê de Ética em Pesquisa.

\section{RESULTADOS E DISCUSSÃO}

No período analisado foram notificados no SINAN - Sistema de Informações de Agravos de Notificação, 1.869 registros de acidentes de trabalho com materiais biológicos por enfermeiras e enfermeiros, sendo a maior frequência encontrada foi no sexo feminino $1.632(87,3 \%)$.

Os anos que ocorreram maiores índices de notificações foram 2017 e 2018 respectivamente $276(14,8 \%)$ e $270(14,4 \%)$. Verificou-se que a maior parte das enfermeiras que sofreram acidente, $1.362(83,5 \%)$ tinha entre 20 a 39 anos, eram de cor parda 972
$(52,0 \%)$ seguido da cor branca 521 $(27,9 \%)$, e entre os enfermeiros a maior frequência encontrada foi de 100 $(42,6 \%)$ para os que tinham idade entre 40 a 59 e $107(45,5 \%)$ para os que tinham de 60 a 79 anos, já a raça/cor foi maior a parda $128(54,5 \%)$, empregados registrados $73(31,1 \%)$ e não trabalhavam em empresa terceirizada 132 (56,2\%), visualizado na (tabela 1).

Quanto ao vínculo de trabalho dos enfermeiros e enfermeira referente à instituição ao qual prestam serviços. O maior número de notificações ocorreu nos empregados registrados 687 $(36,8 \%)$ e em seguida com os servidores públicos estatutários 379 (20,3\%), mas foi possível identificar que de uma forma ou de outra independente do vínculo que tinham com a empresa todos sofreram acidentes com materiais biológicos, e grande maioria desses trabalhadores não trabalhavam em empresa terceirizada 1.025 (54,8\%). Ver na Tabela 1.

Ao analisar as formas pelas quais os enfermeiros obtiveram contato com esses materiais biológicos percebe-se que $1.267(67,8 \%)$ ocorreram através da exposição percutânea e 306 $(16,4 \%)$ foi por exposição de mucosas. Ao verificar o material orgânico do acidente $1.439(77,0 \%)$ foi através do sangue, sendo este o percentual mais elevado. No momento do acidente $1.314(70,3 \%)$ estavam utilizando luvas, e $1.460(78,1 \%)$ a fonte de contaminação era conhecida. Com relação à evolução do caso após a exposição ao material, mais da metade 1.034 $(55,3 \%)$ foi ignorado e $546(29,2 \%)$ seguiu de alta para paciente com fonte negativa. Um quantitativo expressivo de profissionais $1.592(85,2 \%)$ estavam vacinados contra hepatite $B$ e havia $87(4,7 \%)$ não vacinados, 1.040 $(55,6 \%)$ tiveram o exame Anti-HIV 
negativo e $318(17,0 \%)$ não realizaram o teste Anti-HIV, a frequência das variáveis foi igual para ambos os sexos. (Tabela 2).

Com o passar dos anos foi possível observar que os índices de notificação de acidentes com materiais biológicos aumentaram, contudo não houve oscilações muito discrepantes. Possivelmente o aumento pode estar atrelado a maior conscientização da população, quanto a importância da notificação. Porém, pode-se ter muitos casos que ainda não são notificados.

O sexo que apresentou maior frequência, foi o feminino. A enfermagem é uma profissão na qual majoritariamente é composta por mulheres. Isso justifica o elevado percentual de acidentes com profissionais do sexo feminino. Este fato é concernente com os resultados de outro estudo (VALIM et al., 2014). Deve-se pensar também no fato de muitas vezes a mulher desempenha uma dupla jornada de trabalho ficando mais expostas a riscos no processo laboral (OLIVEIRA et al., 2015).

A faixa etária 20 a 39 anos obteve uma elevada porcentagem, retratando que a população feminina jovem é a mais acometida. Entretanto, foi evidenciado que com o avançar da idade esse número reduz consideravelmente. Provavelmente, pelo menor número de pessoas com mais idade desenvolvendo trabalho assistencial.

Ao analisar as variáveis situação no mercado de trabalho e empresa terceirizada, constatou-se a maior parte dos trabalhadores são registrados e não trabalham para empresas terceirizadas. Essas variáveis apresentam fatores importantes que podem potencializar o risco de acidentes de ambiente de trabalho permite que os mesmos fiquem expostos a diversas patologias, por isso, é importante monitorar a situação vacinal, que é considerada medida protetiva, pois ocorre a redução de riscos e aumento da manutenção da saúde do trabalhador (AMORIM et al., 2014; MACHADO et al., 2017). Entretanto, é necessário não somente a vacinação, como também a verificação de fato da imunização por meio de exames sanguíneos.

Tabela 1 - Características sociodemográficas, vínculo de trabalho de enfermeiros e enfermeiras que sofreram acidentes com materiais biológicos na Bahia, no período de 2009 a 2018.

\begin{tabular}{|c|c|c|c|c|c|}
\hline \multirow{2}{*}{$\begin{array}{c}\text { Variáveis } \\
\text { Faixa Etária }\end{array}$} & \multirow{2}{*}{$\frac{\text { Ignorado }}{\mathrm{n}}$} & \multicolumn{2}{|c|}{ Masculino } & \multicolumn{2}{|c|}{ Feminino } \\
\hline & & $\mathrm{n}$ & $\%$ & $\mathrm{n}$ & $\%$ \\
\hline $\begin{array}{l}\text { Dados inconsisten- } \\
\text { tes }\end{array}$ & - & & 0,4 & 27 & 1,6 \\
\hline 20 a 39 anos & 2 & - & - & 1362 & 83,5 \\
\hline 40 a 59 anos & - & 100 & 42,6 & 238 & 14,6 \\
\hline 60 a 79 anos & - & 107 & 45,5 & 5 & 0,3 \\
\hline Raça/Cor & & & & & \\
\hline Ign/Branco & 1 & 23 & 9,8 & 184 & 11,3 \\
\hline Branca & - & 60 & 25,5 & 461 & 28,2 \\
\hline Preta & - & 20 & 8,5 & 129 & 7,9 \\
\hline Amarela & - & 3 & 1,3 & 12 & 0,7 \\
\hline Parda & 1 & 128 & 54,5 & 843 & 51,7 \\
\hline Indígena & - & 1 & 0,4 & 3 & 0,2 \\
\hline $\begin{array}{l}\text { Situação no } \\
\text { mercado de } \\
\text { trabalho }\end{array}$ & & & & & \\
\hline Ign/Branco & - & 18 & 7,7 & 76 & 4,7 \\
\hline $\begin{array}{l}\text { Empregado regis- } \\
\text { trado }\end{array}$ & - & 73 & 31,1 & 612 & 37.5 \\
\hline Empregado & & 20 & & & \\
\hline registrado & - & & 7,7 & 149 & 9,1 \\
\hline Autônomo & - & 2 & 0,9 & 7 & 0,4 \\
\hline Servidor & & 49 & & & \\
\hline Estatutário & - & & 20,9 & 330 & 20,2 \\
\hline Serv. Púb. Celetista & - & 25 & 10,6 & 110 & 6,7 \\
\hline Desempregado & - & - & - & 3 & 0,2 \\
\hline Trab. temporário & - & 13 & 5,5 & 118 & 7,2 \\
\hline Cooperativado & - & 18 & 7,7 & 99 & 6,1 \\
\hline Trab. avulso & - & - & - & 3 & 0,2 \\
\hline Empregador & - & - & - & 1 & 0,1 \\
\hline Outros & - & 17 & 7,2 & 124 & 7,6 \\
\hline $\begin{array}{l}\text { Empresa Terceiri- } \\
\text { zada }\end{array}$ & & & & & \\
\hline Ign/Branco & 1 & 57 & 24,3 & 393 & 24,1 \\
\hline Sim & 1 & 35 & 14,9 & 282 & 17,3 \\
\hline Não & - & 132 & 56,2 & 893 & 54,7 \\
\hline Não se aplica & - & 11 & 4,7 & 64 & 3,9 \\
\hline
\end{tabular}

Dentro da análise dos resultados anti-HIV após a exposição com o material contaminado, percebe-se que muitos resultados foram negativos, mas $17 \%$ dos trabalhadores não realizaram este teste após a exposição com os mesmos. A literatura traz que a realização dos exames sorológicos e testes rápidos devem ser realizados de forma rápida no prazo médio de uma hora para que todas as medidas profilá- ticas aconteçam o mais breve possível conforme preconiza o Ministério da Saúde, tudo isso para se evitar possíveis complicações na vida desses profissionais, e devido ainda à grande frequência desses acontecimentos dentro das instituições de saúde, o estudo ainda aborda a importância da implantação de novos protocolos de assistência dentro das mesmas para melhorar a segurança desses trabalhadores durante o seu trabalho laboral (FERNANDES et al., 2019). Mesmo que encontrado em pequena proporção é necessário a investigação daqueles que não realizaram os testes, para melhor compreender a sua não realização, mesmo conhecendo suas consequências.

Trabalho, pois trabalhadores com vínculos empregatícios precários, muitas vezes, se veem na necessidade de possuir mais de um vínculo de trabalho, o que ocasiona uma rotina acrescida estresse e cansaço (OLIVEIRA et al., 2015).

A maioria dos acidentes com materiais biológicos para ambos os sexos foi através da exposição percutânea, e com menor frequência através da exposição mucosa, envolvendo o sangue como material orgânico de maior contato. Este mesmo resultado foi encontrado em outro estudo realizado com enfermeiros em um hospital de alta complexidade do interior do estado de São Paulo onde os pesquisadores trazem que dentre os fatores associados à exposição ocupacional com esses materiais, $61,5 \%$ foi a exposição percutânea e 79,2\% o sangue como fluido de maior contato (NEGRINHO et al., 2017), e este fator ainda é muito comum entre os profissionais da saúde (FERNANDES et al., 2019).

A maior parte dos profissionais estava utilizando luvas durante o mo- 
mento do acidente, como encontrado também no estudo de Amorim. Isso é um fator positivo, pois demostra que os profissionais estão fazendo uso do equipamento de proteção individual (EPI). Contudo, compreende-se que todos deveriam fazer uso, visto que os EPI são de uso obrigatório. As luvas não impedem que o acidente aconteça, pois podem ser perfuradas, porém, elas podem diminuir o contato da enfermeira com sangue e secreções (AMORIM et al., 2014).

Existe um elevado índice de subnotificação, sobre a evolução dos casos provavelmente em decorrência da falta de informação relativa à sua importância ou até devido a receio por parte do acidentado, ou preenchimento das fichas de notificações de casos de acidentes que chegam à unidade e não são registados. (MUROFUSE, MARZIALE, GEMELLI, 2005).

Dos casos notificados que tiveram alta com fonte sorológica negativa segundo (OLIVEIRA et al., 2015), pode está relacionado através da tomada de medidas corretas de alguns procedimentos de cuidados que ainda se tornam insuficientes em relação à quantidade de pacientes que ignoram o seguimento da evolução dos casos após os acidentes.

Quanto a situação vacinal para Hepatite B, observou-se que grande parte dos profissionais estavam vacinados, como o que é preconizado para profissionais de saúde. $\mathrm{O}$

Os dados do presente estudo realizado pela Revista Brasileira de Enfermagem (2017), constata que a exposição ocupacional a material biológico é um fator preocupante entre os profissionais de enfermagem no ambiente hospitalar. Grande parte dos casos verificados por este mesmo estudo identifica uma taxa de $18,6 \%$ de enfermeiros acometidos por acidentes envolvendo material biológico em um hospital oncológico no estado de São Paulo (NEGRINHO et al., 2017).

Tabela 2 - Características dos acidentes de trabalho em enfermeiros com material biológico, na Bahia, entre os anos de 2009 a 2018.

\begin{tabular}{|c|c|c|c|c|c|}
\hline \multirow{2}{*}{$\begin{array}{ll}\text { Variáveis Ig } \\
\text { Fxnos Percutâne }\end{array}$} & Ignorado & \multicolumn{2}{|c|}{ Masculino } & \multicolumn{2}{|c|}{ Feminino } \\
\hline & $\mathbf{n}$ & n & $\%$ & $\mathbf{n}$ & $\%$ \\
\hline Ign/Branco & 1 & 17 & 7,2 & 149 & 9,1 \\
\hline Sim & - & 168 & 71,5 & 1099 & 67,3 \\
\hline Não & 1 & 50 & 21,3 & 384 & 23,5 \\
\hline \multicolumn{6}{|l|}{ Exposição mucosa } \\
\hline Ign/Branco & 1 & 33 & 14,0 & 286 & 17,5 \\
\hline Sim & - & 36 & 15,3 & 270 & 16,5 \\
\hline Não & 1 & 166 & 70,6 & 1076 & 65,9 \\
\hline \multicolumn{6}{|l|}{ Material Orgânico } \\
\hline Mat. Orgânico & - & 16 & 6,8 & 132 & 8,1 \\
\hline Sangue & - & 184 & 78,3 & 1255 & 76,9 \\
\hline Líqui & - & - & - & 7 & 0,4 \\
\hline o pleural & - & - & - & 3 & 0,2 \\
\hline iniótico & - & - & - & 3 & 0,2 \\
\hline sangue & 1 & 16 & 6,8 & 82 & 5 \\
\hline Sor & - & 1 & 0,4 & 14 & 0,9 \\
\hline Outros & 1 & 18 & 7,7 & 136 & 8,3 \\
\hline \multicolumn{6}{|l|}{ Uso de luva } \\
\hline $\mathrm{Ign} / \mathrm{Br}$ & - & 11 & 4,7 & 94 & 5,8 \\
\hline Sim & 2 & 181 & 77,0 & 1131 & 69,3 \\
\hline Não & - & 43 & 18,3 & 407 & 24,9 \\
\hline \multicolumn{6}{|l|}{ Fonte conhecida } \\
\hline Ign/Branco & - & 11 & 4,7 & 137 & 8,4 \\
\hline Sim & 2 & 190 & 80,9 & 1268 & 77,7 \\
\hline Não & - & 34 & 14,5 & 227 & 13,9 \\
\hline \multicolumn{6}{|l|}{ Evolução caso } \\
\hline Ign/Branco & 2 & 130 & 55,3 & 902 & 55,3 \\
\hline $\begin{array}{l}\text { Alta com conv. } \\
\text { sorológica }\end{array}$ & - & 3 & 1,3 & 21 & 1,3 \\
\hline $\begin{array}{l}\text { Alta sem conv. } \\
\text { sorológica }\end{array}$ & - & 28 & 11,9 & 182 & 11,2 \\
\hline $\begin{array}{l}\text { Alta pac. fonte } \\
\text { negativo }\end{array}$ & - & 68 & 28,9 & 478 & 29,3 \\
\hline Abandono & - & 6 & 2,6 & 49 & 3 \\
\hline Situação & & & & & \\
\hline \multicolumn{6}{|l|}{ Hepatite B } \\
\hline Ign/Branco & - & 31 & 13,2 & 159 & 9,7 \\
\hline Vacinado & 2 & 194 & 82,6 & 1396 & 85,5 \\
\hline Não Vacinado & - & 10 & 4,3 & 77 & 4,7 \\
\hline \multicolumn{6}{|l|}{ Anti-HIV } \\
\hline Ign/Branco & - & 50 & 21,3 & 429 & 26,3 \\
\hline Positiv & - & 1 & 0,4 & 8 & 0,5 \\
\hline Negativo & 2 & 143 & 60,9 & 895 & 54,8 \\
\hline Inconclusivo & - & 2 & 0,9 & 21 & 1,3 \\
\hline Não realizado & - & 39 & 16,6 & 279 & 17,1 \\
\hline
\end{tabular}

Relatos no mesmo estudo identificando exposições ocupacionais percutâneas $(78,8 \%)$ destacam-se sangue visível e agulha oca, sendo esta ultima principal objeto causador de acidentes. Do total de exposições, $25,0 \%$ dos profissionais acidentados não estavam utilizando luvas para procedimentos (NEGRINHO et al., 2017).

O manuseio da matéria orgânica tem relação direta ao exercício da enfermagem nas atividades designadas, sendo elas a administração de medicamentos, coletas de amostras sanguíneas, curativos, higiene corporal e tricotomia, que utiliza lâminas cortantes. Todos esses cuidados predispõem ao contato direto com o sangue. Dessa forma, observou-se no estudo de Amorim que $83,87 \%$ envolveram acidentes com matéria orgânica sanguínea. Essa incidência é redistribuída também aos acidentes com administração de medicação por via subcutânea, reencape de agulhas e descarte inadequado de perfuro cortante (AMORIM et al., 2014).

No que se diz respeito à contaminação por exposição mucosa, durante o contato com profissionais que se acidentaram, foram associados os cuidados que envolviam a via subcutânea e manipulação com agulha de insulina para realização de teste de glicemia capilar. Por se tratar de um simples exame, não há os devidos cuidados necessários para tal procedimento (AMORIM et al., 2014).

Por isso, é necessário que o uso frequente de EPI seja indispensável na prevenção de acidentes, assim como a exposição aos materiais biológicos e validação da segurança entre profissionais e pacientes (NEGRINHO et al., 2017).

\section{CONCLUSÃO}

Com a análise dos dados obtidos observou-se que a maior frequência encontrada foi no sexo feminino, na faixa etária jovem de 20 a 39 anos, na cor parda. O maior número de notificações ocorreu nos empregados registrados, os enfermeiros obtiveram contato com esses materiais biológicos foram através de da exposição percutânea e por exposição de mucosas. Ao verificar o material orgânico do acidente ocorreu através do contato com o sangue. 
Ressaltando que no momento do acidente os mesmos estavam utilizando luvas, grande parte desde profissionais sabiam dos ricos e estavam vacinados contra hepatite $B$. Nesse sentido, se faz necessário maior ênfase na prevenção dos casos de acidentes em todas as áreas, principalmente na área da saúde, tendo em vista os riscos que podem levar o trabalhador a sofrer um acidente de trabalho biológico.

As entidades empregadoras, públicas ou privadas por sua vez, devem investir mais em ações e projetos de educação continuada aos profissionais das áreas de saúde que utilizam qualquer tipo de material que possa causar acidente, incentivando-os nas formas de prevenção.

\section{REFERÊNCIAS}

AMORIM, I. G. et al. Caracterização dos acidentes com exposição a material Biológico envolvendo a equipe de enfermagem de um Hospital universitário. Revista da Universidade Vale do Rio Verde, v. 12, n. 1, p. 811-819, 2014.

CAIXETA R. B., BARBOSABRANCO A. Acidente de trabalho com material biológico, em profissionais de saúde de hospitais públicos do Distrito Federal, Brasil 2002/2003. Caderno de Saúde Pública, v. 21, n. 3, p. 737-746, 2005.

FERNANDES et al. Acidentes laborais e a construção coletiva de um protocolo assistencial. Revista de enfermagem UFPE online. Recife. v. 13, n. 2, p. 511-7, 2019.

MACHADO M. R. M., MACHADO F. D. A. Acidentes com material biológico em trabalhadores de enfermagem do Hospital Geral de Palmas (TO). Revista Brasileira de Saúde Ocupacional, v. 36, n. 124, p. 274281, 2011.
MACHADO et al. Imunidade para Hepatite B entre Trabalhadores de um Hospital de Referência em Doenças Infectocontagiosas, vítimas de acidente com material biológico. Journal of Health and Biological Sciences. v. 5, n. 1, p. 62-70, 2017.

MUROFUSE NT, MARZIALE MHP, GEMELLI LMG. Acidente com material biológico em hospital universitário do oeste do Paraná. Rev Gaúch Enferm. v. 26, n. 2, p. 65-78, 2005.

NEGRINHO et al. Fatores associados à exposição ocupacional com material biológico entre profissionais de enfermagem. Revista Brasileira de Enfermagem. v.70, n.1, jan./fev. 2017

OLIVEIRA, E. C. et al. Análise epidemiológica de acidentes de trabalho com exposição a material biológico entre profissionais de enfermagem. SANARE-Revista de Políticas Públicas, v. 14, n. 1, 2015.

TRINDADE D. M. et al. Exposição a materiais biológicos/ Ministério da Saúde, Secretaria de Atenção à Saúde, Departamento de Ações Programáticas Estratégicas. Editora do Ministério da Saúde, p.5-14, 2006.

VALIM, M. D. et al. Ocorrência de acidentes de trabalho com material biológico potencialmente contaminado em enfermeiros. Acta Paulista de Enfermagem, v. 27, n. 3, p. 280-286, 2014. 